\title{
A Review on the Orthoplastic Approach to Lower Limb Reconstruction
}

\author{
${ }^{1}$ Division of Plastic and Reconstructive Surgery, Department of \\ Surgery, University of Utah School of Medicine, Salt Lake City, \\ Utah, United States \\ ${ }^{2}$ Department of Orthopaedic Surgery, Rambam Health Care \\ Campus, Haifa, Israel \\ ${ }^{3}$ Department of Orthopaedic Surgery, University of Pennsylvania \\ Perelman School of Medicine, Philadelphia, Pennsylvania, \\ United States \\ ${ }^{4}$ Division of Plastic Surgery, University of Pennsylvania Perelman \\ School of Medicine, Philadelphia, Pennsylvania, United States
}

Shaun D. Mendenhall ${ }^{1} \quad$ Oded Ben-Amotz ${ }^{2} \quad$ Rikesh A. Gandhi ${ }^{3} \quad$ L. Scott Levin ${ }^{3,4}$

\author{
Address for correspondence L. Scott Levin, MD, FACS, Penn \\ Musculoskeletal Center, 3737 Market Street, 6th FI, Philadelphia, PA \\ 19104, United States (e-mail: scott.levin@pennmedicine.uphs.edu).
}

Indian J Plast Surg 2019;52:17-25

\begin{abstract}
Keywords

- orthoplastic

- open fracture

- limb salvage

- soft tissue coverage

- reconstructive microsurgery

- soft tissue reconstruction

- bone reconstruction

Just as in the craft of carpentry, a stable foundation and framework are absolutely essential to the final function of a building, but no more important than the drywall, trim, and paint that make the building functional, durable, and livable. Reconstruction of the lower extremity is similar; the orthopaedic surgeon must obtain stable fixation of the damaged or diseased bone once a thorough debridement of nonviable bone is performed, while the plastic or orthopaedic soft tissue surgeon must provide vascularized, stable coverage. These two components are complementary and both contribute to the success or failure of functional limb restoration. The stability of bone repair will predict the ultimate functional status, while the vascularized envelope will enhance the biology of bone and soft tissue healing. When both components are properly attended to, the result is often a functional limb with an acceptable appearance. While a single surgeon need not perform both of these tasks (although some may choose to do so), the orthopaedic and plastic surgeon involved in this care must have a clear understanding of each other's role and their importance for a good outcome. This is what we call the orthoplastic approach to reconstructive surgery of the extremities, that is, the application of principles and practice of both specialties applied simultaneously to optimize the outcomes in limb reconstruction. In this review article, we discuss the history of orthoplastic surgery, the key elements of orthoplastic surgery, and thoughts on factors that lead to good outcomes through select cases.
\end{abstract}

\section{Introduction}

\section{The History of Orthoplastic Surgery}

The beginnings of orthoplastic surgery came in the days before surgical specialization when individuals such as Gaspar Tagliacozzi, Velpau, Ambroise Pare, Dupuytren, and Malgaigne built surgical careers treating composite defects all over the body. These master surgeons became the founding fathers of both orthopaedic and plastic surgery. One of the earliest modern orthopaedic and plastic surgery collaborations was between W. Arbuthnot Lane and Sir Harold Gillies in 1919. Lane, an orthopaedic surgeon, wrote the preface for Major Gillies' textbook, and so began the modern era of orthoplastic surgery. ${ }^{1,2}$

Further development of the orthoplastic concept came with the advent of reconstructive microsurgery. Starting over a century ago with Alexis Carrel's description of an end-to-end vascular anastomosis in 1902, the discipline of microvascular surgery was born. ${ }^{3}$ Another key advancement that led to the development of microvascular surgery was the description 
of using an operative microscope to perform a microvascular anastomosis by Jacobson and Suarez in $1960 .{ }^{4}$ Once these fundamentals of vascular surgery were in place, advancements in microsurgical instrumentation, sutures, and needles led to the beginnings of the modern microsurgical era. ${ }^{5}$

With the fundamentals of microsurgery in place, the concept of orthoplastic limb salvage surgery further evolved. In 1968, Susumu Tamai reported the first successful digital replantation. ${ }^{5}$ From this era until today, innovations have continued including widespread use of muscle, fasciocutaneous, perforator, and vascularized bone flaps, all which have become paramount in the salvage of the traumatized extremities. Success in microsurgery and replantation led to the development of vascularized composite allotransplantation, including hand and face transplants, over the past 20 years as the highest rung on the reconstructive ladder. ${ }^{6,7}$ Combined, these microsurgical advancements have become one of the pillars of orthoplastic surgery.

\section{The Orthoplastic Approach}

The combination of the strengths of both orthopaedic surgery and plastic surgery constitutes the modern definition of orthoplastic surgery:

"The principles and practices of both specialties applied to clinical problems simultaneously, either by a single provider, or team of providers, working in concert for the benefit of the patient."2,8-11

This combined orthoplastic approach to patients with severe injuries to the lower extremities or patients with oncologic processes requiring lower limb salvage will not only lead to better outcomes such as quicker time to bone union, more durable soft tissue coverage, less pain, and better function, but also less complications, shorter hospital stays, and higher patient satisfaction which are all important in the current healthcare climate of bundled payments and outcomes-based reimbursement. ${ }^{12}$ Trauma center designation has led to improved management of open fractures. ${ }^{13}$ The orthoplastic approach necessitates a dedicated team of an orthopaedic surgeon and a reconstructive microsurgeon readily available on a weekly basis to address open fractures necessitating soft tissue coverage. The orthopaedic surgeon should consult the reconstructive microsurgeon prior to or during initial stabilization of an open fracture to ensure all members agree with the course of treatment. Arrangements should be made for the reconstructive surgeon to evaluate the soft tissue injury either intraoperatively at the time of the index stabilization or immediately postoperatively. A dedicated weekly orthoplastic operating room (OR) has shown to positively impact the care of such patients. ${ }^{14}$ An intensive care unit that has experiencing managing postoperative surgical patients is necessary to monitor the patient following the procedure.

This review article will focus on key elements of the orthoplastic approach to lower extremity reconstruction including a thorough evaluation of the extremity, the role and timing of debridement and flap coverage, the key elements of bone fixation, limb salvage versus amputation, and common flaps used in orthoplastic surgery.

\section{Orthoplastic Evaluation of the Injured Lower Extremity}

Combined bone and soft tissue injuries to the lower extremity come in many different shapes, locations, and sizes. The Gustilo classification has become the most widely used method to classify open fractures of the lower extremity. ${ }^{15,16}$ This classification consists of three main categories of open fractures with three subtypes (-Table 1 ).

Other key elements of the orthoplastic evaluation of the traumatized lower extremity include ${ }^{8}$ :

Table 1 The Gustilo grading system of open tibial fibular fractures and treatment ${ }^{12,13}$

\begin{tabular}{|l|l|l|}
\hline Gustilo grade & Description & Treatment \\
\hline I & Open fracture, with clean wound $<1 \mathrm{~cm}$ in length & $\begin{array}{l}\text { Irrigation, debridement, ORIF/EF, } \\
\text { primary closure }\end{array}$ \\
\hline II & $\begin{array}{l}\text { Open fracture, with wound }>1 \mathrm{~cm} \text { but }<10 \mathrm{~cm} \text { in length with- } \\
\text { out extensive soft-tissue damage, loss, flaps, or avulsions }\end{array}$ & $\begin{array}{l}\text { Irrigation, debridement, ORIF/EF, } \\
\text { primary closure }\end{array}$ \\
\hline III & $\begin{array}{l}\text { Open fracture with extensive soft-tissue lacerations }(>10 \mathrm{~cm}), \\
\text { damage, or loss or an open segmental fracture. Subcategorized } \\
\text { as below: }\end{array}$ & Variable, see below \\
\hline IIIA & $\begin{array}{l}\text { Adequate soft tissue coverage of the fractured bone despite } \\
\text { extensive soft-tissue laceration or flaps, or high-energy trauma } \\
\text { irrespective of wound size }\end{array}$ & $\begin{array}{l}\text { Irrigation, debridement, ORIF/ } \\
\text { EFrimary closure or sometimes } \\
\text { requires STSG or local soft tissue flap } \\
\text { coverage }\end{array}$ \\
\hline IIIB & $\begin{array}{l}\text { Extensive soft-tissue injury with periosteal stripping and bone } \\
\text { exposure. Usually associated with major contamination }\end{array}$ & $\begin{array}{l}\text { Irrigation, debridement, ORIF/EF, } \\
\text { often requires free tissue transfer or } \\
\text { local muscle flaps or perforator based } \\
\text { flaps }\end{array}$ \\
\hline IIIC & $\begin{array}{l}\text { Open fracture associated with an arterial injury requiring repair, } \\
\text { irrespective of degree of soft-tissue injury }\end{array}$ & $\begin{array}{l}\text { Irrigation, debridement, ORIF/EF, } \\
\text { vascular repair, often requires free } \\
\text { tissue transfer or local muscle flaps } \\
\text { or perforator based flaps }\end{array}$ \\
\hline
\end{tabular}


- Timing and environment of injury

- Mechanism and amount of energy absorption

- Fracture configuration and amount of bone loss

- Systemic conditions, medical status, other associated injuries

- Extent of surrounding soft tissue injury, shearing, loss (including integument, muscle, fascia, and periosteum)

- Vascularity, sensibility, and motor function of the limb

- Possibility of compartment syndrome

Location of soft tissue injuries along the axis of the leg is also an important factor to analyze. Traditionally, the traumatized lower extremity has been divided in to thirds in an algorithmic manner to help guide treatment:

- Upper one-third: gastrocnemius pedicled flaps

- Middle one-third: hemi-soleus pedicled flaps

- Lower one-third: free tissue transfer

However, with modern-day microsurgical techniques including perforator based local flaps such as propeller flaps and reverse flow flaps, and improvement in free tissue transfers, many options now exist for each region of the extremity. A more modern approach to analyzing a lower extremity defect is to focus on the nature of the defect, what's missing and what needs to be replaced or reconstructed, and what local or distant options exist for achievement of pain-free weight-bearing ambulation. However, vital to the success of any reconstruction is the process of preparing the wound by thorough debridement.

While this review focuses primarily on reconstructing the traumatized lower extremity, similar principles apply to lower extremity limb salvage procedures for oncologic conditions or chronic osteomyelitis involving the bones of the lower extremity.

\section{Orthoplastic Wound Preparation}

In our practice, the following principles of irrigation, debridement, and coverage have proven successful:

- Get the patient to the OR as soon as reasonably possible (considering vascular status, level of contamination, concomitant injuries, etc.)

- Perform a "radical necrectomy," treating the wound as a "pseudotumor" removing all devitalized soft tissue, bone, and surface contaminated tissue sharply with a scalpel

- If unhappy with the wound's appearance (continued bacterial colonization, purulent appearance, or ongoing necrotic or fibrinous debris) take the patient back for a second or third look

- Irrigation with normal saline at low pressure

- Use of a hydrosurgery device for surface decontamination, minor debridement, or removal of granulation tissue prior to skin grafting

- Keep wound moist at all times utilizing a "wet to wet" dressing with petrolatum gauze covered with saline moist gauze

- Negative pressure wound therapy can keep the wound clean and buy more time

- Early coverage desirable
- Manage edema

- Prevent equinus contracture

(-Fig. 1) demonstrates a representative case of an 8-yearold child with a Gustilo $3 \mathrm{C}$ injury to the lower extremity where these principles were applied. The orthoplastic approach led to successful limb salvage and a good outcome for the child (-Fig. 1).

\section{Basic Principles of Bone Fixation}

Open fractures with soft tissue loss require coordinated care to achieve bony union, guard against infection, and maximize functional outcomes. The Arbetigemenschaft fur Osteosythesfragen group (AO) developed four basic principles for surgical treatment of fractures. These include:

1. Fracture reduction and fixation to restore anatomical relationships

2. Fracture fixation providing absolute or relative stability as the fracture, patient and injury requires

3. Preservation of blood supply to soft tissues and bone by gentle reduction techniques and careful handling

4. Early mobilization and rehabilitation of the injured part and patient as a whole

The development of these principles emphasized the importance of soft tissue coverage, and has led to the awareness that successful management of major orthopaedic trauma is inseparable from careful management of the soft tissue envelope.

Mutilating extremity injuries often involve significant bony comminution and contamination, which require temporary external fixation and repeat debridements. If the bone contamination is significant or there is concern for ongoing infection, fractures require external fixation as the definitive treatment until bone healing. Important soft tissue reconstruction principles must be considered with placement of external fixation pins and bars as follows:

- Pins should be as far from the wound as possible while maintaining fracture stability.

- Bars should not obstruct the soft tissue window around the zone of injury.

- In the leg, mid anterior pins are better than medial or lateral pins to allow for vascular access for free flaps and rotational muscle and perforator flaps.

- The frame must be easily loosened for adjustments needed during reconstruction.

Conversion from external fixation to plates or intramedullary nails in open fractures that have been thoroughly debrided in combination with immediate soft tissue coverage has proven effective in the treatment of severe extremity trauma ( - Fig. 1)..$^{1718}$ This approach is ideal when possible and allows for reconstruction to proceed without interference of external fixator devices.

It is imperative that both the orthopaedic surgeon and the reconstructive plastic surgeon be familiar with the needs and approaches of the other to optimize the functional outcome for the patient. This orthoplastic approach to limb salvage 

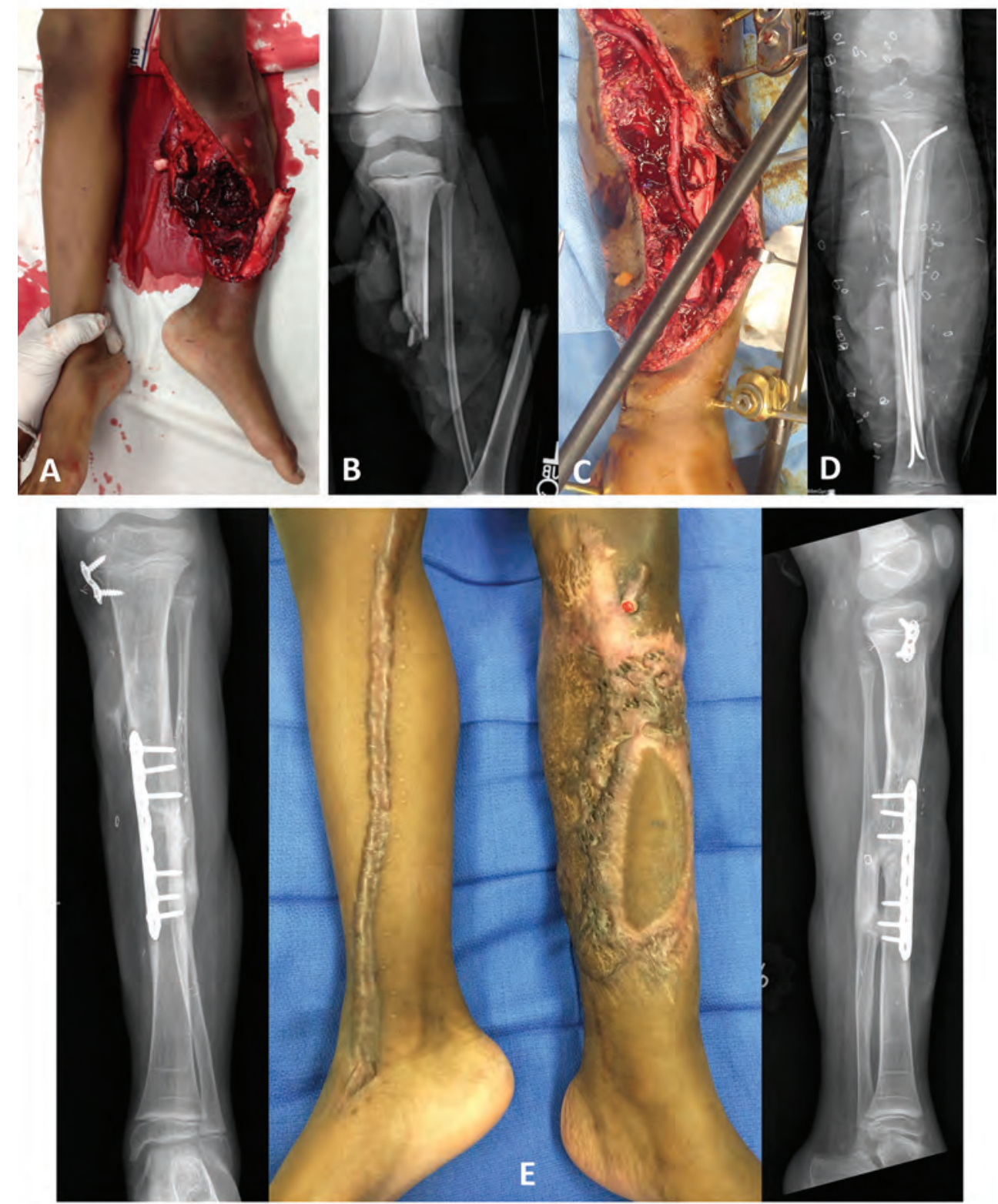

Fig. 1 Orthoplastic approach to Gustilo 3C injury in a child. A 8-year-old child with a severe limb-threatening injury after being struck by a car (A, B). This required immediate stabilization with an external fixator and revascularization with a reversed saphenous vein graft (C). After multiple debridements, conversion to internal fixation and free latissimus myocutaneous flap reconstruction was performed (D). Although the patient developed hypertrophic scaring and required revision open reduction internal fixation with bone grafting, he went on to union with an acceptable outcome (E).

will maximize outcomes, although at times amputation must be considered before embarking on the often lengthy limb salvage journey. ${ }^{2}$

\section{Limb Salvage versus Amputation}

Several studies have been performed in attempt to determine factors predicting success of limb salvage. The largest of these was a subset of the Lower Extremity Assessment Project (LEAP) study, which evaluated 556 patients by using five different injury severity-scoring systems. None of the indicators were useful to help differentiate between limbs that necessitated amputation versus those that could be successfully salvaged. The authors did find that that low scores could be useful to predict limb-salvage potential; however, the converse was not true. The authors concluded the low sensitivity of the indices failed to support the validity of the scores as predictors of amputation. ${ }^{19} \mathrm{~A}$ separate study examined seven lower extremity injury severity indices and found none were predictive of ultimate functional outcome at 2 years. ${ }^{20}$ The LEAP study group investigated the outcome of limb salvage versus reconstruction and found patients who underwent limb salvage versus primary amputation had no difference in long-term outcomes and both cohorts were found to have less than ideal outcomes. ${ }^{21}$ Importantly, the LEAP study demonstrated that overall function was affected by a patient's economic, social, and emotional recourses more than by treatment course. Factors associated with poor outcomes included poverty, lack of health insurance, poor 
social network, involvement in litigation, nonwhite race, and smoking. ${ }^{21}$

Given this information and the lack of accurate prediction of outcomes with scoring systems, the decision to reconstruct or amputate must often be made based on the judgment of the multidisciplinary orthoplastic team with input from the patient. In our practice, factors that encourage amputation include a mangled lower extremity and:

- Prolonged warm ischemia time ( $>3$ hours)

- Severe muscle damage

- Multilevel injury including ipsilateral tibia and foot crush injury

- Proximal or severe tibial nerve injury that would preclude plantar sensation even with repair

- Poor psychosocial support and coping mechanisms

- Concomitant medical condition or injuries that put patient at surgical significant risk

If amputation is to be performed, the orthoplastic surgeon should attempt to preserve sufficient length and sensibility for effective prosthetic use and utilize the principles of sparepart surgery, fillet flaps, island pedicle flaps, and free flaps as needed. ${ }^{2}$

\section{Commonly Used Flaps in Orthoplastic Reconstruction of the Lower Limb}

Orthoplastic principles can be applied in a variety of reconstructive options. One of the most exciting aspects of orthoplastic surgery is the combination of bone healing principles and microvascular surgery to fill large bone defects after trauma, avascular osteonecrosis, tumor extirpation, or osteomyelitis. Microsurgery has revolutionized the treatment of large segmental bone defects through the use of vascularized bone transfer. A discussion of all free flaps used in orthoplastic surgery is beyond the scope of this review, but below we highlight our two most commonly used flaps.

\section{Free Fibula Osteocutaneous Flap}

Since its description by Taylor et al in 1975, the vascularized fibula bone flap has been used extensively due to its size, acceptable donor site morbidity, and predictable dissection. ${ }^{22}$ Free fibula bone grafts have been utilized for segmental defects of the upper and lower extremity and have a pivotal role in traumatic reconstruction..$^{23}$ We commonly use free fibula grafts anytime a long strut of bone is needed for extremity reconstruction, typically longer than $4 \mathrm{~cm}$. They can be harvested with a moderate size skin paddle and/or part of the soleus or gastrocnemius muscles when composite tissue reconstruction is needed. We often take a small skin paddle for monitoring purposes even if soft tissue reconstruction is not needed.

Technical pearls of raising a free fibula osteocutaneous flap include:

- Mark the length of the fibula, and measure $7 \mathrm{~cm}$ of bone proximally and distally to preserve to protect the common peroneal nerve and maintain ankle stability, respectively ( - Fig. 2A)
- Incise directly over the middle of the fibula from the fibular neck to the distal fibula

- Raise the crural fascia over the peroneal muscles from anterior to posterior

- In the distal third region of the fibula, look for septocutaneous perforators going behind the fibula ( - Fig. 2B) and design the skin paddle based on these perforators (-Fig. 2C)

- Reflect the peroneal muscles anteriorly off the fibula, leaving a $3 \mathrm{~mm}$ cuff of muscle on the bone to protect the periosteum

- Find and protect the common peroneal nerve at the proximal extent of the incision

- Incise the anterior intermuscular septum and sweep the anterior compartment muscles off the interosseus membrane

- Carefully incise the interosseus membrane, and make proximal and distal osteotomies

- Use a bone clamp to roll the fibula posteriorly, locate the pedicle distally and ligate

- Dissect the flap from distal to proximal, carefully taking down any small branches coming from the pedicle

- Leave a small cuff of flexor hallucis longus and soleus on flap to protect periosteum

- Follow pedicle as close to tibioperoneal trunk as possible to maximize pedicle length

- After the flap is harvested ( - Fig. 2D), the flexor hallucis longus is resuspended, and a layered closure is performed over a drain (Fig. 2E).

\section{Free Medial Femoral Condyle Flap}

Free vascularized bone grafts from the medial femoral condyle are increasing in versatility and popularity. The medial femoral condyle flap or medial genicular artery flap as a source of vascularized bone transfer has had a surge in use in a variety of bony reconstructions. It has been utilized with increased frequency for larger bone grafting procedures, and has become a reliable alternative to free fibular grafts for intermediate sized osseous defects up to $\sim 4 \mathrm{~cm}$ in greatest dimention. ${ }^{24,25,26}$ The flap has more recently been described as a method of supplying well-vascularized corticoperiosteum as a method of treating recalcitrant nonunions all over the body including the foot/ankle, tibia, femur, scaphoid, radius, ulna, humerus, and clavicle. ${ }^{27}$ We have found this to be a versatile flap with reliable anatomy, with a wide array of orthoplastic applications.

Technical pearls of raising a free medial femoral condyle osteocutaneous flap include:

- Mark out the distal femur, proximal tibia, knee joint, and medial collateral ligament of the knee ( - Fig. $\mathbf{3 A}$ )

- The incision is made toward the posterior border of the femur extending across the medial femoral condyle

- Dissection is carried down to the vastus medialis fascia which is incised for a subfascial dissection

- The vastus medialis is retracted anteriorly which reveals the dominant descending geniculate pedicle running along the femur and paralleling the adductor tendon (-Fig. 3B) 

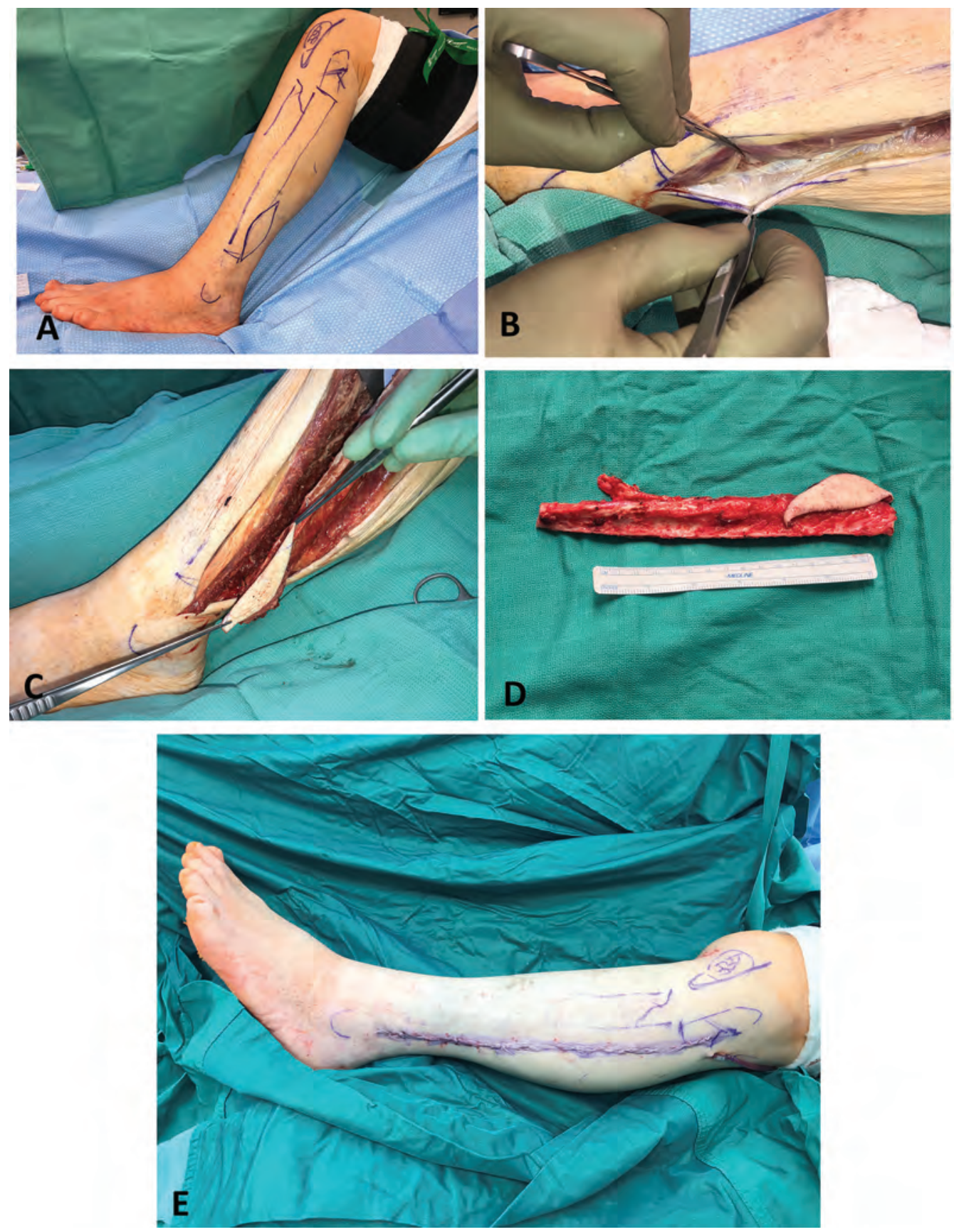

Fig. 2 Raising a free fibula osteocutaneous free flap. Initial skin markings preserving $7 \mathrm{~cm}$ of proximal and distal fibula (A). In a subfascial plane, skin perforators can be located at the posterior border of the fibula along its distal one-third (B). The skin paddle is designed based on the perforators located (C). A cuff of muscle is left on the fibula to protect its periosteum (D). The flexor hallucis longus is resuspended and the skin is then closed over a drain (E).

- If the pedicle is difficult to locate here, dissect over the face of the medial femoral condyle where the pedicle fans out, and trace it back proximally

- Once the pedicle is located, it is followed proximally to the take-off of the superficial femoral artery

- Take note of any cutaneous and muscle branches coming off the artery during the dissection as these can be followed and used as a chimeric flap if needed

- Cautery is used to incise the periosteum for the bone flap over the medial femoral condyle
- An oscillating saw and/or osteotomes are then used to raise the bone flap, talking care to protect the pedicle proximally

- The pedicle is then carefully lifted up with a swath of periosteum

- At this point, the nondominant pedicle of the medial superior geniculate artery is often encountered heading toward the popliteal fossa. This is clipped and ligated, or used as a lifeboat if the descending genicular artery is not of sufficient size ( $\mathbf{- F i g . ~ 3 B )}$ ). 

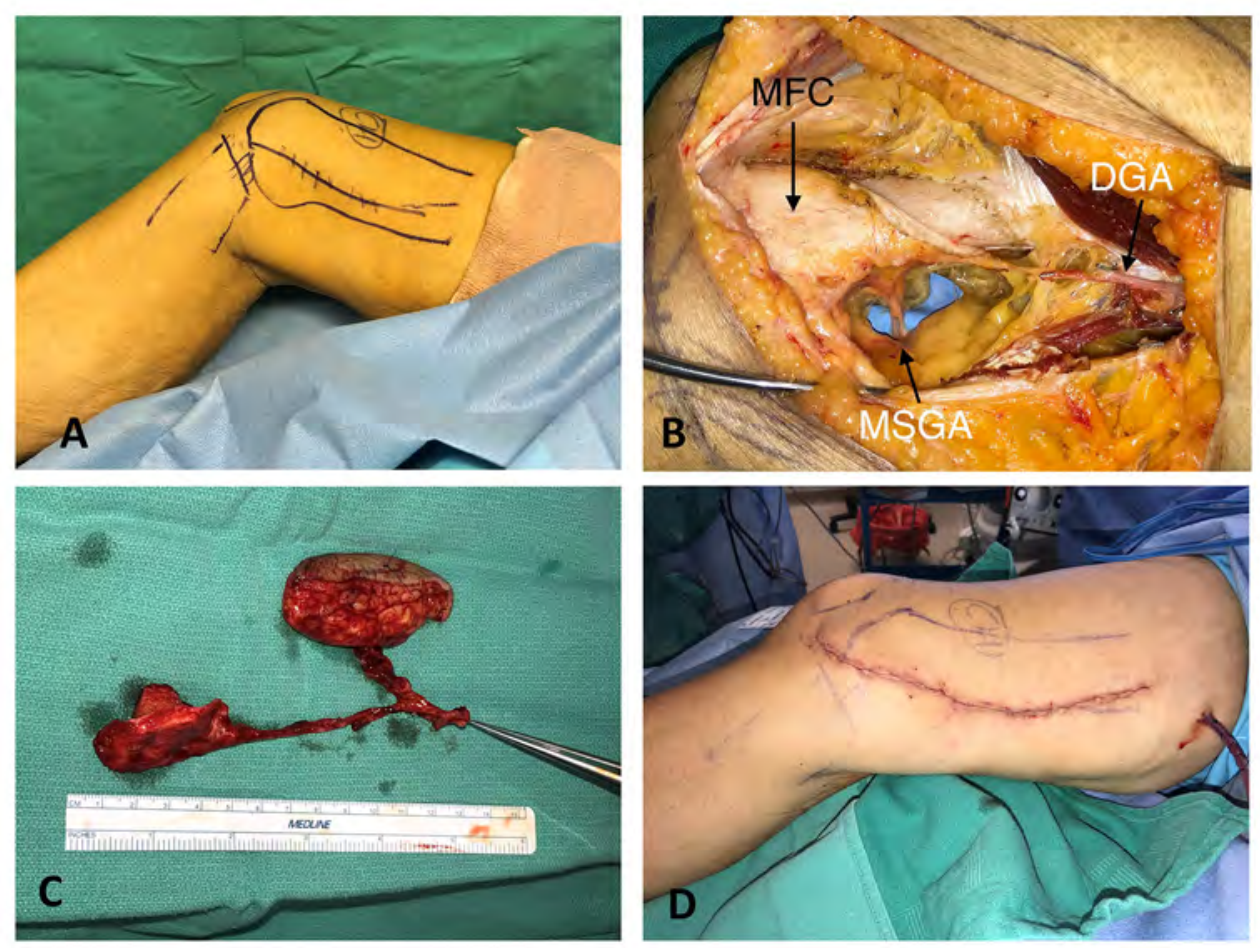

Fig. 3 Raising a medial femoral condyle osteocutaneous free flap. Panel (A) shows the preoperative marking of the distal femur, proximal tibia, the medial collateral ligament, and the incision toward the posterior border of the femur. Panel (B) shows the vascular anatomy of the medial femoral condyle (MFC) flap including the usual dominant pedicle the descending geniculate artery (DGA) and lifeboat nondominant pedicle the medial superior geniculate artery (MSGA). Panel (C) shows the osteocutaneous flap and panel (D) shows closure over a drain.

- The flap is harvested ( - Fig. $\mathbf{3 C}$ ) and Gelfoam and thrombin are then placed in the bone defect and the leg is closed over a drain ( - Fig. 3D)

\section{Summary/Conclusion}

The concept of the orthoplastic approach brings together the strengths of orthopaedic surgery of stable bone reconstruction and rehabilitation with the microvascular soft tissue and aesthetic principles of plastic surgery simultaneously to maximize outcomes in extremity reconstruction. When both perspectives are clearly understood and applied, outcomes in reconstruction and salvage are maximized. Key aspects of orthoplastics include building a foundation of effective debridement and stable fracture fixation followed by well-vascularized soft tissue coverage that together bring success in treating composite injuries of the lower extremity.

\section{Clinical Case \#1}

A 38-year-old male was involved in a head-on collision motor vehicle accident and suffered severe bilateral lower extremity trauma. On the right leg, he had a Gustilo IIIA open fracture of the tibia with $15 \mathrm{~cm}$ of bone loss ( - Fig. 4A). His fractures were treated at an outside hospital which consisted of an external fixator and a large antibiotic spacer for the tibial defect. One month after the accident he was referred to our institution for limb salvage. He was then taken to the OR for free fibula flap to reconstruction of the tibial defect. Although fractured, the ipsilateral fibula was used because the contralateral fibula also had a fracture and previous open reduction internal fixation (ORIF). There was some difficulty raising the fibula flap because of the significant trauma to the area, but the pedicle and proximal bone was well preserved ( - Fig. 4B). The fibula was slid down into the bone spacer cavity using the same approach from the fibula harvest. This was placed into a slot in the tibia and secured with a Steinmann pin, a small spring plate, and the external fixator was replaced ( - Fig. 4C, D, E). The peroneal pedicle of the fibula was anastomosed to the anterior tibial vessels in an end-to-side fashion. The patient did well postoperative and was taken back to the OR 6 weeks later for placement of a Taylor Spatial Frame (TSF) and arthrodesis of the fibula to the talus ( $\mathbf{- F i g}$. $\mathbf{4 F}$ ). He was then transitioned to toe touch weight bearing 2 weeks later, and weight bearing as tolerated 4 weeks after that. Once the fibula hypertrophies sufficiently, the TSF will be removed. He has had one pin site infection postoperatively that was treated successfully with IV antibiotics.

\section{Clinical Case \#2}

A 14-year-old high level cyclist presented with 3 months of left anterior knee pain and swelling. He was noted on radiographs to have a sclerotic lesion in his proximal tibia ( - Fig. 5A and B). A biopsy revealed a proximal tibial osteosarcoma. After neoadjuvant chemotherapy, we underwent left proximal tibia radical resection with reconstruction using an intercalary allograft and a vascularized free fibula. The physis was able to be spared using this technique. 


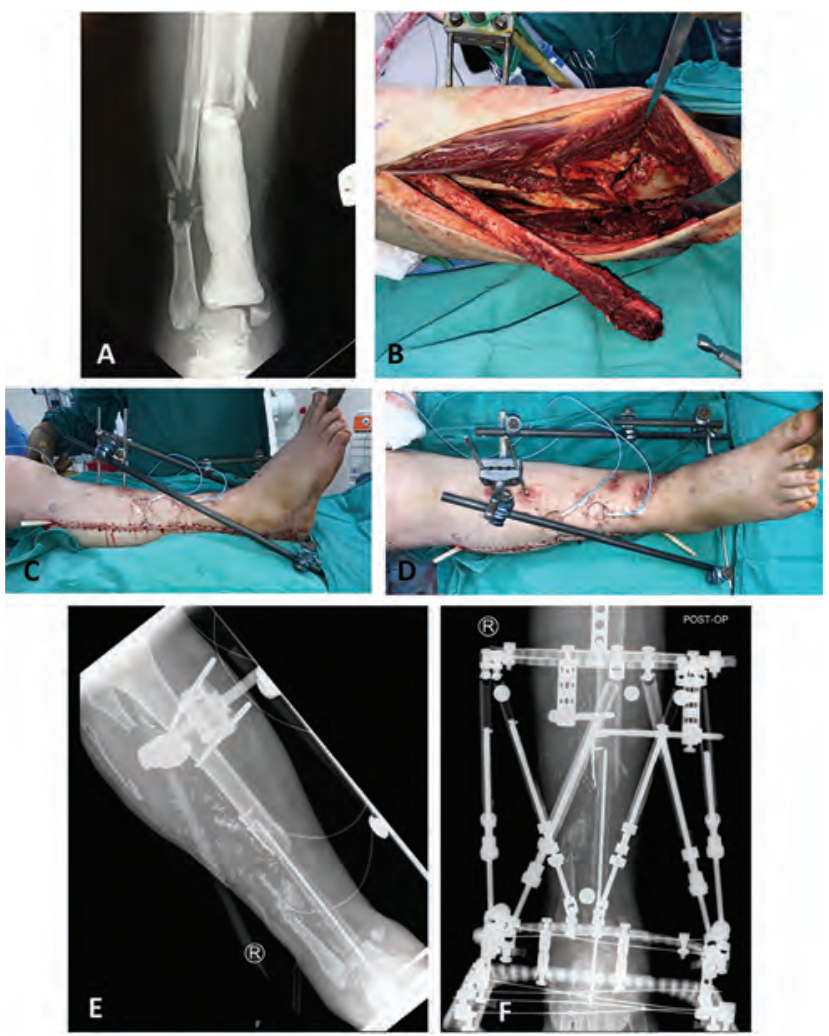

Fig. 4 Clinical case of orthoplastic reconstruction of a $15 \mathrm{~cm}$ tibial defect after a Gustilo 3A tib/fib fracture (A). Ipsilateral free fibula reconstruction was performed for limb salvage (B). Panels (C) and (D) show the immediate postoperative appearance of the leg with a delta external fixator in place. Panel $(\mathbf{E})$ shows the immediate postoperative X-ray and panel (F) shows the Taylor Spatial Frame that was placed after 6 weeks to allow gradual weight bearing and hypertrophy of the free fibula.

The vascularized fibular graft is thought to benefit the healing of the allograft. The graft was harvested from the contralateral limb along with the peroneal artery and anastomosed to the posterior tibial artery and vein in the operative limb. Postoperative radiographs demonstrated an appropriate reconstruction (-Fig. 5C). The patient was advanced to weight bearing as tolerated at 12 weeks and brought back to the OR at this time to remove the proximal transphyseal screws. Radiographs at this time demonstrated callus at the distal end of the allograft and host-bone interface ( - Fig. 5D). For tumor cases, a radical resection of the bone tumor and reconstruction with an intercalary allograft with vascularized bone graft have been shown to be a successful and durable option.

\section{Clinical Case \#3}

A 76-year-old male suffered from severe post-traumatic tibiotalar ankle arthritis after an injury skydiving. He presented with partial talar avascular necrosis with

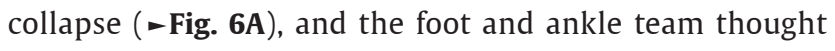
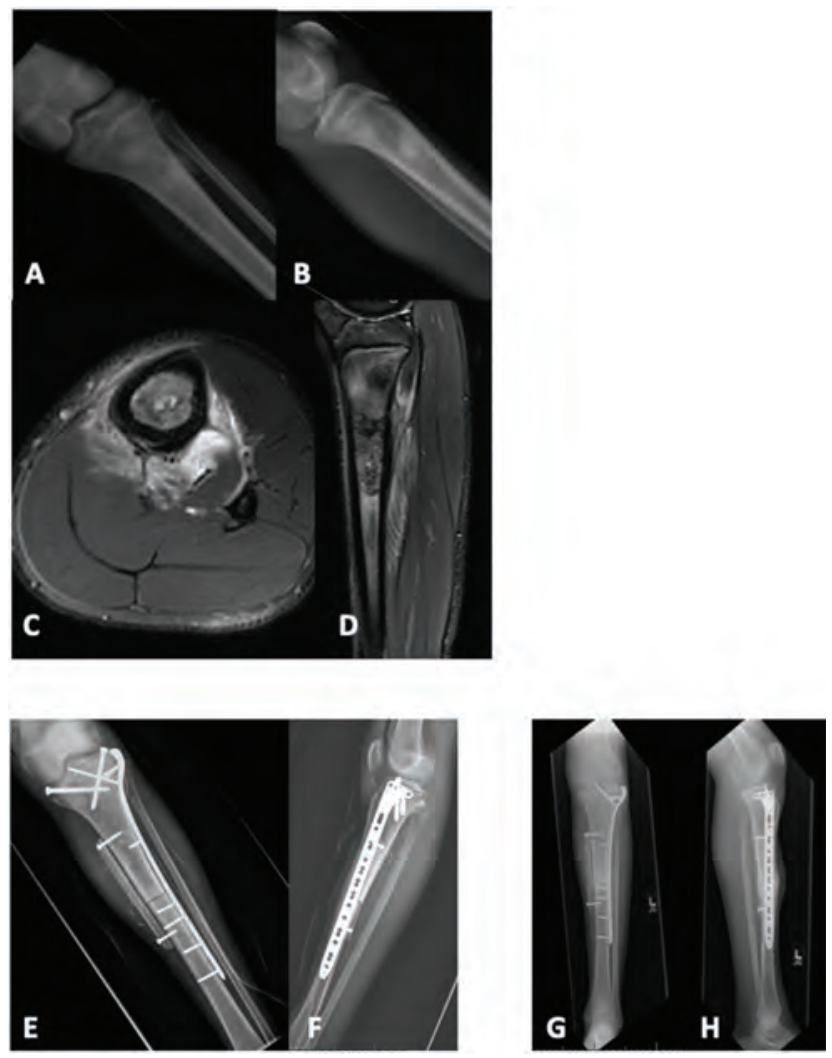

Fig. 5 Clinical case of a vascularized free fibula bone graft for a tibial osteosarcoma intercalary allograft reconstruction. A 14-year-old male with a left proximal tibial osteosarcoma on radiograph (A, B) and magnetic resonance imaging (C, D). Reconstruction was performed using a long locking plate and intercalary allograft with a vascularized free fibula (E, F). A 3 months follow-up demonstrated adequate healing with good callus formation at the distal allografthost bone interface $(\mathbf{G}, \mathbf{H})$.

he could benefit from a vascularized medial femoral condyle flap at the time of tibiotalar fusion to augment bone healing. The foot and ankle team resected the dead bone of the tibia and talus, and fused the joint with cannulated screws, leaving an anterior bone defect for the bone flap ( $\boldsymbol{- \text { Fig. }} \mathbf{6 B}$ ). Under tourniquet control, the anterior tibial vessels were located just proximal to the ankle, and a medial femoral condyle osteocutaneous flap was raised as described above ( $\boldsymbol{- \text { Fig. }} \mathbf{6 C}$ ). The bone flap was impacted in to the defect and secured with a single 0.045 Kirschner wire. Microvascular anastomosis was then performed end-to-end to the anterior tibial vessels with excellent blood flow to the flap ( - Fig. 6D, E). He recovered postoperatively and was discharged home. He had one small bout of cellulitis that was treated successfully with a short course of IV antibiotics followed by oral antibiotics. At 8 weeks postoperatively, he was allowed to progress to full weight bearing in a cam boot, which was discontinued at 11 weeks. He has fully healed the fusion and is able to walk un-assisted ( - Fig. 6F). 

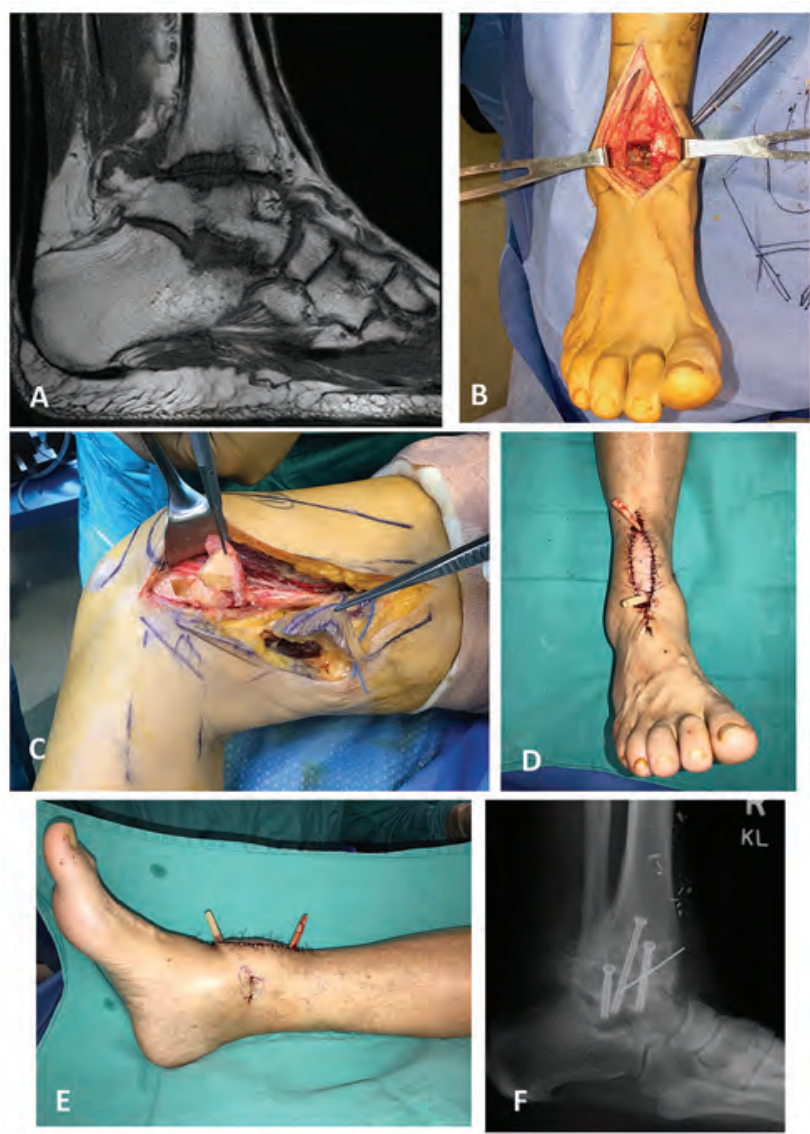

Fig. 6 Clinical case of a medial femoral condyle free osteocutaneous flap for a tibiotalar fusion. A 76-year-old male with severe tibiotalar post-traumatic arthritis with partial collapse of the talar dome from avascular necrosis as demonstrated on $\mathrm{T} 1$ magnetic resonance imaging scan (A). Fusion was performed by debriding the avascular necrosis site and placing cannulated screws across the joint, which left a bone defect (B). A medial femoral condyle osteocutaneous flap was performed to augment bone healing $(\mathbf{C}-\mathbf{F})$. Panel $(\mathbf{F})$ shows 4 months follow-up with good consolidation of bone at the fusion site and around the bone flap.

\section{Disclosures and Conflicts of Interest}

The authors do not have any financial relationships or conflicts of interest related to this study to disclose.

\section{References}

1 Levin LS. Reconstructing Soft Tissue Defects of the Foot and Ankle: The Orthoplastic Approach. 2012; https://www.aofas. org/PRC/meeting/Documents/ReconstructingSoftTissueD.pdf. Accessed July 25, 2018

2 Lerman OZ, Kovach SJ, Levin LS. The respective roles of plastic and orthopedic surgery in limb salvage. Plast Reconstr Surg 2011;127(Suppl 1):215S-227S

3 Carrel D. [Operative Technic Of Vascular Anastomoses And Visceral Transplantation]. Lyon Med 1964;212:1561-1568

4 Jacobson JH, Suarez E. Microsurgery in anastomosis of small vessels. Paper presented at: Surgical forum; 1960

5 Tamai S. History of microsurgery. Plast Reconstr Surg 2009;124(6, Suppl):e282-e294

6 Shores JT, Malek V, Lee WPA, Brandacher G. Outcomes after hand and upper extremity transplantation. J Mater Sci Mater Med 2017;28(5):72
7 Sosin M, Rodriguez ED. The face transplantation update: 2016. Plast Reconstr Surg 2016;137(6):1841-1850

8 Levin LS. The reconstructive ladder. An orthoplastic approach. Orthop Clin North Am 1993;24(3):393-409

9 Levin SL. Orthoplastic reconstruction of the hand. Curr Opin Orthop 1993;4(4):10-13

10 Heitmann C, Levin LS. The orthoplastic approach for management of the severely traumatized foot and ankle. J Trauma 2003;54(2):379-390

11 Tintle SM, Levin LS. The reconstructive microsurgery ladder in orthopaedics. Injury 2013;44(3):376-385

12 Boriani F, Ul Haq A, Baldini T, et al. Orthoplastic surgical collaboration is required to optimise the treatment of severe limb injuries: a multi-centre, prospective cohort study. J Plast Reconstr Aesthet Surg 2017;70(6):715-722

13 Stammers J, Williams D, Hunter J, Vesely M, Nielsen D. The impact of trauma centre designation on open tibial fracture management. Ann R Coll Surg Engl 2013;95(3):184-187

14 Fernandez MA, Wallis K, Venus M, Skillman J, Young J, Costa ML. The impact of a dedicated orthoplastic operating list on time to soft tissue coverage of open lower limb fractures. Ann R Coll Surg Engl 2015;97(6):456-459

15 Gustilo RB, Mendoza RM, Williams DN. Problems in the management of type III (severe) open fractures: a new classification of type III open fractures. J Trauma 1984;24(8):742-746

16 Gustilo RB, Anderson JT. Prevention of infection in the treatment of one thousand and twenty-five open fractures of long bones: retrospective and prospective analyses. J Bone Joint Surg Am 1976;58(4):453-458

17 Gopal S, Majumder S, Batchelor AG, Knight SL, De Boer P, Smith RM. Fix and flap: the radical orthopaedic and plastic treatment of severe open fractures of the tibia. J Bone Joint Surg $\mathrm{Br}$ 2000;82(7):959-966

18 Bhandari M, Guyatt GH, Swiontkowski MF, Schemitsch EH. Treatment of open fractures of the shaft of the tibia. J Bone Joint Surg Br 2001;83(1):62-68

19 Bosse MJ, MacKenzie EJ, Kellam JF, et al. A prospective evaluation of the clinical utility of the lower-extremity injuryseverity scores. J Bone Joint Surg Am 2001;83-A(1):3-14

20 Ly TV, Travison TG, Castillo RC, Bosse MJ, MacKenzie EJ; LEAP Study Group. Ability of lower-extremity injury severity scores to predict functional outcome after limb salvage. J Bone Joint Surg Am 2008;90(8):1738-1743

21 MacKenzie EJ, Bosse MJ. Factors influencing outcome following limb-threatening lower limb trauma: lessons learned from the Lower Extremity Assessment Project (LEAP). J Am Acad Orthop Surg 2006;14(10 Spec No.):S205-S210

22 Taylor GI, Miller GD, Ham FJ. The free vascularized bone graft. A clinical extension of microvascular techniques. Plast Reconstr Surg 1975;55(5):533-544

23 Jupiter JB, Gerhard HJ, Guerrero J, Nunley JA, Levin LS. Treatment of segmental defects of the radius with use of the vascularized osteoseptocutaneous fibular autogenous graft. J Bone Joint Surg Am 1997;79(4):542-550

24 Hayashi A, Maruyama Y. The medial genicular artery flap. Ann Plast Surg 1990;25(3):174-180

25 Sakai K, Doi K, Kawai S. Free vascularized thin corticoperiosteal graft. Plast Reconstr Surg 1991;87(2):290-298

26 Kazmers NH, Thibaudeau S, Steinberger Z, Levin LS. Upper and lower extremity reconstructive applications utilizing free flaps from the medial genicular arterial system: a systematic review. Microsurgery 201;8;38(3):328-343

27 Deng AD, Innocenti M, Arora R, Gabl M, Tang JB. Vascularized small-bone transfers for fracture nonunion and bony defects. Clin Plast Surg 2017;44(2):267-285 